\title{
Exploiter un corpus de données textuelles sans post-traitement : l'écriture burlesque de la Fronde
}

Exploiting a Textual Corpus without Post-processing: the Burlesque Writing of the Fronde

Karine Abiven, Jean-Baptiste Tanguy et Gaël Lejeune

\section{OpenEdition}

\section{Journals}

Édition électronique

URL : https://journals.openedition.org/revuehn/2355

DOI : 10.4000/revuehn.2355

ISSN : 2736-2337

Éditeur

Humanistica

\section{Référence électronique}

Karine Abiven, Jean-Baptiste Tanguy et Gaël Lejeune, «Exploiter un corpus de données textuelles sans post-traitement : l'écriture burlesque de la Fronde », Humanités numériques [En ligne], 4 | 2021, mis en ligne le 01 décembre 2021, consulté le 29 novembre 2022. URL : http://

journals.openedition.org/revuehn/2355; DOI : https://doi.org/10.4000/revuehn.2355

Creative Commons - Attribution 4.0 International - CC BY 4.0 https://creativecommons.org/licenses/by/4.0/ 


\title{
humanités numériques
}

\section{Exploiter un corpus de données textuelles sans post-traitement : l'écriture burlesque de la Fronde}

\author{
Exploiting a Textual Corpus without Post- \\ processing: the Burlesque Writing of the Fronde
}

Karine Abiven, Jean-Baptiste Tanguy et Gaël Lejeune

\section{Résumés}

Les « mazarinades " burlesques de la Fronde sont des écrits bien connus des littéraires et des historiens mais peu étudiés systématiquement, en raison notamment de leur difficulté d'accès et de constitution en corpus. Nous cherchons d'abord à délimiter ce corpus et à définir des corpus contrastifs propres à révéler les spécificités de l'écriture burlesque dans ce contexte politique. Ensuite, nous abordons le problème de l'acquisition des données textuelles et nous exposons le processus d'océrisation d'imprimés anciens et son évaluation. Puis, nous choisissons d'exploiter ces données brutes, sans traitement, ce qui semble bien permettre d'obtenir des résultats (dans des domaines tels que le lexique, la métrique ou l'énonciation), en employant différentes méthodes (fouille textométrique et observation des $n$-grammes de caractères). Nous montrons ainsi la spécificité, dans l'écriture burlesque de la Fronde, de certains motifs littéraires et de certaines rimes. Il s'agit donc ici de présenter l'approche d'un corpus dans toutes les phases du processus : depuis la sélection des données, leur acquisition, leur constitution en corpus, jusqu'à leur exploitation statistique et algorithmique et l'interprétation de cette fouille.

The texts printed during the Fronde ("mazarinades") and written in "burlesque" style are a set of documents well known to literary and historical scholars but little studied in a systematical way, mainly because they are difficult to access and turn into a corpus. Our first task here is to delimit this corpus and to define contrastive corpora in order to re- 
veal the specificities of "burlesque writing" in this political context. Then, the problem of acquiring textual data arises: the process of digitizing old printed matter and its evaluation are thus exposed. We choose to exploit these raw data, without post-processing or text correction, which still seems to allow us to obtain results (regarding lexicon, metre or enunciation), with various methods (textometry and observation of character n-grams). We thus show the specificity, in the burlesque writing of the Fronde, of some literary motifs or rhymes. The aim is therefore to present the approach of a corpus in all the steps of the process: from the selection of data, their acquisition, and their transformation into a corpus, to their statistical and algorithmic exploitation, and their interpretation through text mining.

\section{Entrées d'index}

MOTS-CLÉS : littérature, numérisation, constitution de corpus, acquisition de données, analyse textuelle, stylistique

KEYWORDS: literature, digitisation, corpus building, data acquisition, textual analysis, stylistics

\section{Des données au corpus}

L'examen instrumenté des faits de langue et de style sur corpus connaît depuis quelques années des développements aussi bien épistémologiques qu'empiriques, dans des travaux insistant notamment sur le paramètre du genre (Rastier 2011 ; Novakova et Siepmann 2020). Dans notre cas, il s'agit d'explorer un ensemble d'écrits dits " burlesques ${ }^{1}$ ", pris parmi les brefs imprimés parus en France lors de la Fronde (16481653) et traditionnellement appelés " mazarinades ". On peut estimer à plusieurs centaines (parmi les quelque 5500 écrits imprimés pendant la Fronde) les pièces relevant de cette pratique d'écriture, caractérisée par le mélange des registres et le jeu avec les normes, qui s'exerce alors surtout en vers dans des poésies en octosyllabes. Si cette masse d'écrits s'avère en pratique difficile à lire de près ${ }^{2}$, il est tentant d'éloigner un peu la focale et de chercher, grâce à des outils statistiques, une autre échelle pour faire émerger des caractéristiques, notamment métriques et lexicales. On a pu présenter ces écrits suscités par la Fronde comme parangons de l'écriture burlesque ${ }^{3}$ : si c'est une réalité au plan éditorial, d'ailleurs difficile à chiffrer ${ }^{4}$, il reste à montrer la spécificité de cette pratique d'écriture poétique en temps de crise politique. L'accès à ces textes, dispersés, et dont il n'existe pas de liste bibliographique moderne, a constitué jusqu'ici un obstacle majeur à une recherche à la fois qualitative et quantitative sur le sujet ${ }^{5}$. C'est en constituant une liste de titres exploitables en format numérique et en regroupant les données librement disponibles à ce jour pour les constituer en corpus de mazarinades burlesques que nous entendons poser les jalons d'une telle étude. 
Cette ambition se heurte toutefois à des problèmes à la fois théoriques et pratiques. D'abord, l'approche sérielle d'écrits d'actualité fait courir le risque de les isoler de leurs contextes de production alors qu'ils ne sont interprétables que lorsqu'ils sont précisément situés (Jouhaud 2009 [1985]). Un repérage purement lexical qui conduirait à l'interprétation d'isotopies ou de thématiques semble hasardeux, en l'absence de regroupements en séries cohérentes de textes (par exemple, relatives à un même parti ou camp politique) : "à décomposer ainsi les discours [par thèmes], on finit par ne plus rencontrer qu'un immense entrelacs d'éléments de sens dispersés sur toute l'étendue d'une aire culturelle, par effacer toute discontinuité ", écrit Maingueneau (1983, 18).

Sur les mazarinades en particulier, qui ne sauraient être prises dans leur ensemble pour des textes "d'opinion ", l'analyse quantitative du lexique a déjà été récusée de manière convaincante (Jouhaud 2009, 39, 13), du moins selon nous tant qu'on ne peut constituer au préalable des sous-corpus sélectionnés voire enrichis. La sélection d'un corpus et le retour aux contextes des occurrences, pour chercher à affiner leurs coordonnées énonciatives premières, apparaîtront ainsi comme un complément nécessaire à la première approche statistique, rejoignant la dernière étape, préconisée en linguistique de corpus, de validation de l'interprétation par le texte ${ }^{6}$. Ensuite se pose le problème de l'acquisition des données : puisque l'intégralité du corpus des mazarinades burlesques n'est pas accessible numériquement, nous en avons construit un échantillon, en choisissant d'exploiter des données ni traitées ni enrichies, mais directement sorties du processus d'océrisation ${ }^{7}$. Or, l'océrisation, consistant en une transcription automatique des données textuelles contenues dans une image numérique, génère nécessairement du bruit ${ }^{8}$. À quel point peut-on se fier à une transcription automatique, même lorsqu'on dispose, comme c'est le cas, d'outils de reconnaissance en partie calibrés pour cet état de langue ? Cette question de la qualité des données se pose ici à la fois pour le corpus d'étude et les corpus contrastifs qu'il faut également constituer pour cerner la spécificité des mazarinades burlesques. Cette réflexion se situe donc à l'articulation entre l'acquisition automatisée des données textuelles par OCR et la pensée de leur usage en corpus.

Entre data paper et exemples applicatifs, il s'agit à la fois d'exposer des données et de prouver par quelques conclusions stylistiques que leur qualité inégale n'est pas un obstacle à leur exploration et qu'elle n'interdit pas de penser concomitamment leur possible amélioration. 


\section{À la recherche des traits d'écriture burlesque}

Entre 1648 et 1653, la production imprimée " en vers burlesques " est un véritable phénomène éditorial, de sorte que les mazarinades sont parfois réduites à ce "style ". Réciproquement, le style burlesque a pu être vu comme une nouveauté des décennies entourant la Fronde, alors qu'il est aujourd'hui admis qu'il est à cette époque "un nouveau nom pour une vieille chose ${ }^{9}$ ". Caractérisée par la discordance entre le référent et le signifiant, l'écriture burlesque l'est aussi par sa visée souvent dite ludique, parfois railleuse (car fondée sur la dérision de tout sans distinction ni privilège, notamment pour le pouvoir en place), et critique $^{10}$. Ajoutons que dans les années 1630-1640 où elle connaît une première éclosion en France, une de ses formes privilégiées est le " travestissement " d'épopée : le burlesque est alors une des formes de la parodie (Genette 1982). Les écrits burlesques de la Fronde recourraient en fait assez peu à ce procédé (Nédélec 2004), ce que nous tenterons de vérifier.

$\mathrm{Au}$ reste, ce "genre d'écrire " est réputé indéfinissable, de sorte qu’il est souvent décrit plutôt en extension, c'est-à-dire par ses manifestations concrètes ${ }^{11}$, qu'en intension, par ses traits définitoires. Signalons que Bar (1960) et Nédélec (2004) ont déjà bien défini cette pratique au sujet $d u$ lexique, l'écriture burlesque étant réputée innover lexicalement ${ }^{12}$. Leurs conclusions principales concernent l'introduction de néologismes et l'usage, peu admis à l'époque, de toutes les variations $\mathrm{du}$ français (diachronique, diatopique et diastratique : jargons techniques, tours populaires et argot, principalement). S'agissant plus précisément des écrits burlesques suscités par la Fronde, ils sont réputés relever d'un style particulièrement cru et obscène ; cette idée est toutefois mise en doute par Nédélec $(2004,385)$, qui souligne que cette variation n'est nullement propre au burlesque. Il serait ainsi intéressant de mesurer la spécificité des écrits de la Fronde en la matière, de même que d'essayer d'extraire les néologismes (point limite du travail, on le verra).

Ces acquis stylistiques et littéraires ont été jusqu'ici obtenus par des intuitions appuyées sur l'analyse d'échantillons, par induction à partir de «quelques coups de sonde " (Nédélec 2004, 432-437), méthode nécessaire quand le terrain empirique est si problématique d'accès. La méthode statistique ici adoptée permet, grâce à un corpus relativement important et sélectionné, la vérification de ces hypothèses confrontées à la réalité des données (approche corpus-based [Pincemin 1999]). 


\section{Constituer un corpus d'écrits burlesques de la Fronde}

\section{Lister des titres : des bibliographies papier à leurs numérisations}

Si aucune liste exhaustive recensant l'ensemble des mazarinades n'existe, des bibliographies de référence ${ }^{13}$ permettent d'atteindre précisément 5059 titres $^{14}$. Ces recueils bibliographiques n'étant pas disponibles en format numérique, nous les avons océrisés en utilisant le logiciel Kraken ${ }^{15}$ dans sa version 2.0.8 (Kiessling 2019). L'ensemble de ces données textuelles a ensuite été structuré afin de pouvoir les utiliser dans un tableur : à chaque titre (et son numéro interne dans le recueil bibliographique) sont associés une date et un lieu d'édition (quand ces données sont disponibles), un nombre de pages ainsi que l'ensemble de la notice donnée dans la bibliographie Moreau ${ }^{16}$. Signalons d'emblée que cette méthode présente le biais de se fonder sur des bibliographies anciennes, qui demandent à être revues ${ }^{17}$ : la nécessité a fait loi en loccurrence.

Parmi ces 5059 titres donc, seuls ceux comportant "burlesque " - ou une variante graphique de ce terme ( lefque ») - sont rassemblés pour constituer notre corpus. Supposer qu'une pièce est burlesque si son titre l'indique peut sembler accorder une confiance excessive aux étiquettes endogènes. Mais la dimension commerciale de ces écrits (peu coûteux, parfois même distribués dans la rue) implique souvent un étiquetage explicite ; le vers burlesque était, à une époque où la vente de petits imprimés sauvait bien des imprimeries, un argument de vente de choix (Jouhaud 2009, 27-28). Le sous-titre en vers burlesques est si fréquent qu'il a fallu ôter les faux positifs liés au fait que le titre ne renvoie pas au contenu, mais seulement à l'octosyllabe, ce mètre étant presque systématique dans l'écriture burlesque - procédé utilisé au moins deux fois ${ }^{18}$, sans doute pour ces raisons commerciales. Pour pallier cette fois le silence induit par cette méthode, un repérage manuel a permis d'ajouter quelques pièces dépourvues de titraille explicite $^{19}$.

Cette liste s'élève, en l'état, à 250 titres $^{20}$, dont 179 sont librement disponibles au téléchargement en ligne. Les plateformes Gallica ${ }^{21}$ et Google Livres ${ }^{22}$ ont été utilisées, avec une priorité pour Gallica qui propose des images et des métadonnées de meilleure qualité. Les numérisations disponibles sur Google Livres proviennent de diverses entreprises de numérisation sans que leurs conditions et protocoles soient accessibles.

Nous présentons deux exemples de problèmes de numérisation observés dans un même document La Nappe renversée, chez Renard, en vers burlesques (Paris, 1649), téléchargées sur Google Livres ${ }^{23}$. La page 6 (figure 1) est très difficilement lisible car on y devine par transparence la page suivante dont l'encre a déteint ; le phénomène est fréquent pour des imprimés furtifs comme les mazarinades, faits sur du papier fin et de mauvaise qualité. La page 12 (figure 2) ne documente que la présence humaine nécessaire pour numériser! 


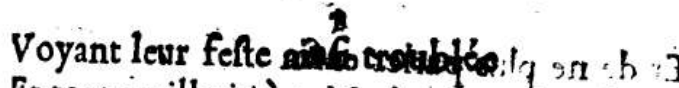

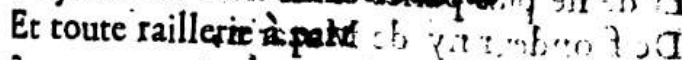

se voyant pris cheziva Rionets

Dont ils nuturent pas la fineffe,

Pleins de colere, toideidurneffec

Je ne s'eftuspasibionls repûs:

Inragez d'eftre interrompus

'ar Meffieurs de la'frondétic

Oont ils auoient fait raillerie

ien allerent ie ne fçay où

1 Chaliot ou à fainct Clou,

Jù mefme à Paris la grand ville

Qui de braues foldats fourmille -

le noftre Roy bons feruiteurs

li'Amiens appelle frondeurs)

efolus de fermer la porte,

'u d'auoir toufiours grande efcorte

ors quils mangeroient chez Renard

it-ce le matin ou le tard,

lais pour moy dedans ce burlefque

noftre braue foldatefque

veux donner vne leçọn

ui fera voir que i'ay raifon,

eft de perdre toute memoire

u. de deffaite ou de victoire

indant tous nos troubles paffez

ì nous faifions lesinfenfez

e bannir de nos conferences

ant de badines medifances

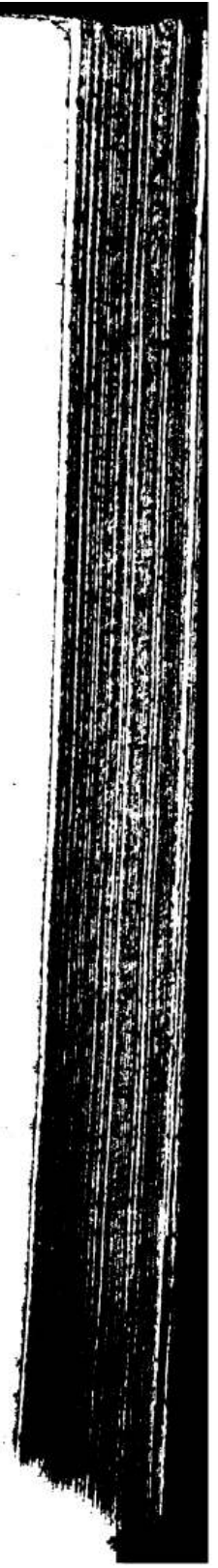




$$
\text { I'I' }
$$




\section{Océrisation}

Distribuées au format PDF, les numérisations ont été converties - en utilisant la librairie pdftoppm (version 0.62.0) - en une suite d'images PNG permettant par la suite de procéder à l'OCR. Un tri parmi l'ensemble des images résultantes a écarté les pages générées par les plateformes (celles qui précisent les conditions générales d'utilisation). Ces images ont ensuite été binarisées, segmentées et océrisées en utilisant Kraken et un modèle d'OCR entraîné pour le français du XVII ${ }^{\mathrm{e}}$ siècle $^{24}$ (Gabay, Clérice et Reul 2020). On dispose finalement de 2822 images PNG et du même nombre de sorties d'OCR ${ }^{25}$ au format HTML, format privilégié au seul texte brut pour les métadonnées qu'il permet d'encoder.

Il convient de préciser d'entrée de jeu que l'océrisation de tels documents historiques est sujette au bruit ${ }^{26}$ (ajouts, suppression ou substitution de caractères), et bien plus que pour les documents nés numériques.

\section{Évaluation des sorties d'OCR}

Classiquement, le taux d'erreur au caractère (ou Character Error Rate, contracté ci-après en CER) est utilisé pour évaluer une sortie d'OCR ; cette mesure supervisée nécessite des transcriptions de référence, manuelles par exemple, dont le présent projet ne dispose pas encore. Aussi privilégions-nous pour le moment des méthodes d'évaluation non supervisées, pouvant suivre plusieurs voies :

- Taux de confiance : Springmann, Fink et Schulz (2016) proposent d'exploiter les taux de confiance disponibles dans les métadonnées des fichiers de sortie (pour Kraken, les fichiers HTML) fournis par les logiciels d'OCR.

- Ressources lexicales : Springmann, Fink et Schulz (2016) explorent une autre piste en cherchant, pour chaque mot de la sortie d'OCR, la distance de Levenshtein le séparant de sa forme moderne la plus proche.

- Bounding boxes : Gupta et al. (2015) utilisent les rectangles résultant de la segmentation de la page pour calculer la proportion de rectangles représentant du bruit.

- Modèle de langue : Tanguy (2020) entraîne des modèles de langue au grain caractère sur un corpus de référence puis récupère les probabilités d'apparition des caractères de la sortie d'OCR.

Nous proposons ici deux adaptations des méthodes proposées par Springmann, Fink et Schulz (2016), ne nécessitant pas l'apprentissage d'un modèle connexe : la moyenne des taux de confiance et le taux de lexicalité ${ }^{27}$. On désignera désormais par $T_{\text {con }}$ la première, qui calcule la moyenne, pour une page, des taux de confiance donnés par le logiciel d'OCR pour chaque caractère. Springmann, Fink et Schulz (2016) ajoutent que " more importantly, all characters with a confidence above average (0.93) are correct ". Une page ayant une moyenne de taux de confiance au caractère supérieure à la moyenne devrait donc être bien océrisée. On nommera $T_{l e x}$ la seconde mesure, qui calcule, pour une page, la proportion de mots ${ }^{28}$ présents dans la sortie d'OCR appartenant 
à un lexique. Le lexique utilisé pour procéder à ce calcul est le LGeRM (Souvay et Pierrel 2009) qui compte plus de trois millions de formes fléchies. Cette mesure est ici invoquée pour savoir si, globalement, les mots présents dans les sorties d'OCR figurent sous une forme attestée.

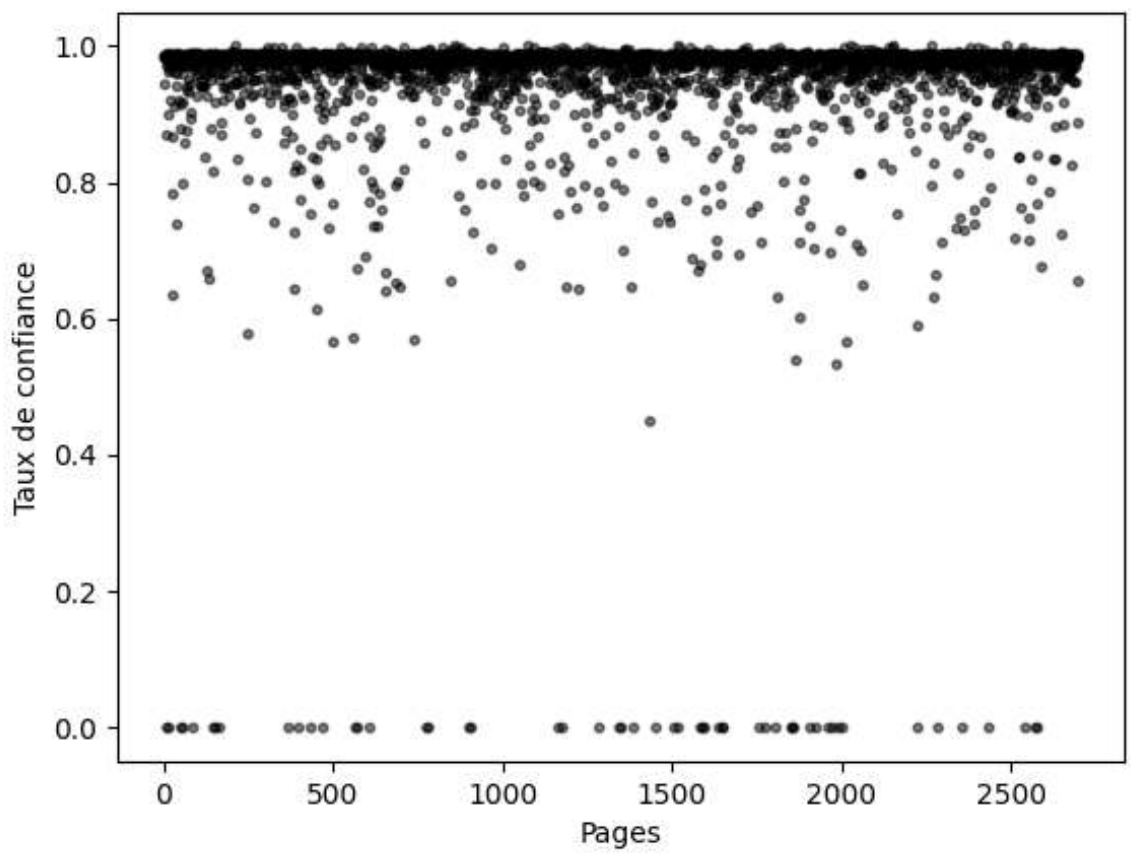

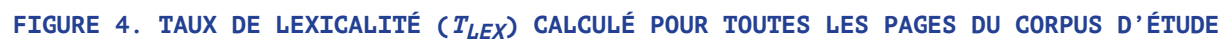

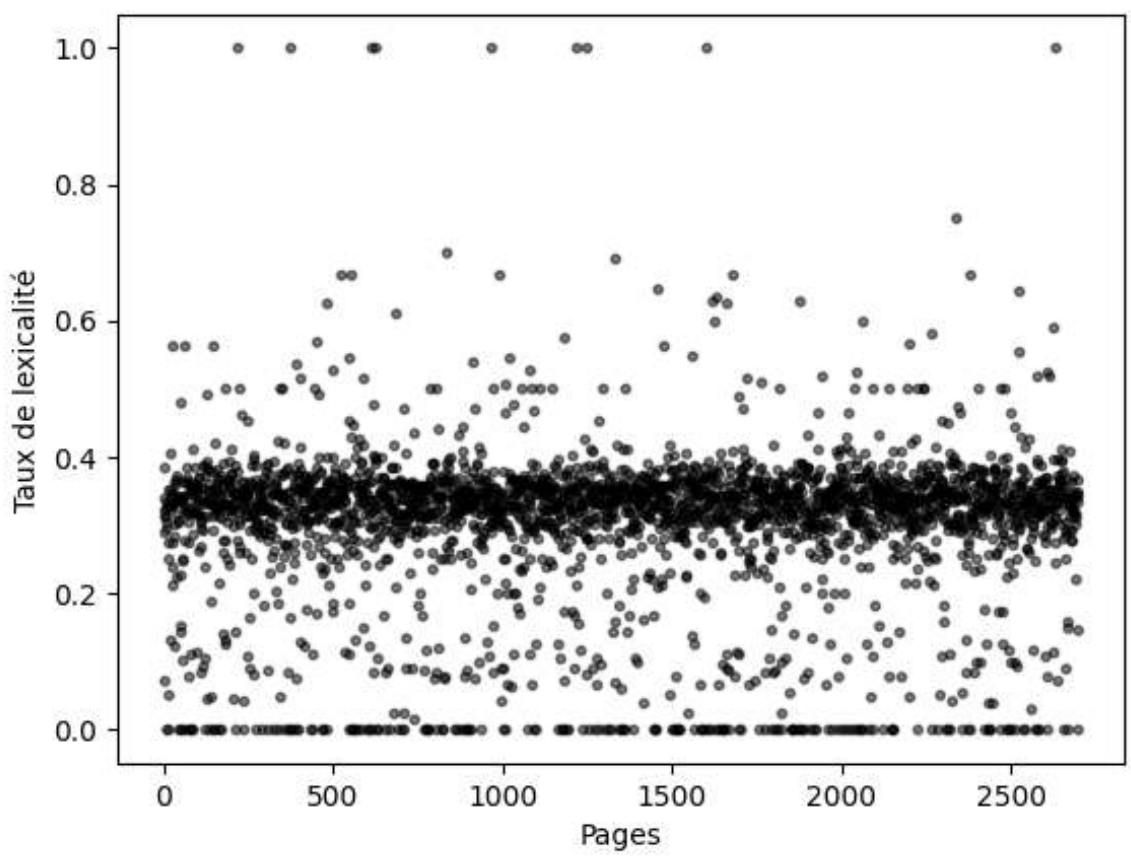


Les figures 3 et 4 montrent que les taux de confiance sont globalement supérieurs au seuil donné par Springmann, Fink et Schulz (2016) - en moyenne, le $T_{\text {con }}$ est égal à 0,98 -, ce qui suggère une qualité au moins correcte des données océrisées ${ }^{29}$. Les taux de lexicalité, quant à eux, nuancent le précédent propos car ils gravitent autour de o,4 pour l'ensemble des pages ; un peu moins de la moitié des mots proposés par Kraken appartiennent au LGeRM. Si l'on peut considérer que les données textuelles océrisées dont nous disposons sont de plutôt bonne qualité, les erreurs semblent être présentes dans l'ensemble du corpus. En effet, un taux de confiance plus faible couplé à un taux de lexicalité plus élevé aurait suggéré une concentration des erreurs dans des zones restreintes du corpus.

Sont proposés ci-dessous en exemple des extraits de deux pages du corpus - l'une ayant été " bien » océrisée selon la mesure $T_{\text {con }}$ et l'autre, «mal» océrisée.

La quatorzième page de L'On du temps tout nouveau, en vers burlesque (sic), par C. D. B. M., avec un $\mathrm{T}_{\text {con }}$ égal à o,98 :

Ce procedé n'eft pas Chreftien,

Il fert à chafcun d'entretien;

Si quelque interefle l'approuue,

ON, auec les fages l'improuue.

La cinquième page de la Réponse au Parlement burlesque de Pontoise, ou l'Anti-burlesque du sieur D. L. R. Parisien, contre l'auteur du libelle intitulé : le Parlement burlesque de Pontoise, avec un $\mathrm{T}_{\text {con }}$ égal à o,64 :

irdeife feiefeueuenle ieefon leuea $\neg$

Sdue oel onu e lue e $\mathrm{n}$ euen lde dae eille $\neg$ pne noue eoeoneune e lieon feedoae ls oereue eenfe ne ur ae e e lenea peeeneeet $\neg$

Un tel bruit peut faire émerger de fausses hypothèses, en particulier dans une étude s'intéressant de près au lexique et à ses innovations (ou néologismes). Par exemple, l'occurrence "bougrene " repérée dans un des textes n'est pas un mot-valise agglomérant habilement la "gangrène " au " bougre " suggérant ainsi l'envahissement inévitable de la France par le "goût italien " ! Il s'agit simplement d'une confusion entre les graphèmes " $r i$ » et " $n$ ». Un retour à l'image du livre numérisé permet de lire «bougrerie». 


\section{Taux de reconnaissance estimé (Gallica)}

La plateforme Gallica propose, pour certaines de ses numérisations, une transcription automatique obtenue par OCR. À cette sortie d'OCR est associé un " taux de reconnaissance estimé ", lequel correspond au pourcentage de mots supposés corrects par le logiciel d'OCR - ce taux est proposé à léchelle du document en tant que moyenne des taux de reconnaissance par page. "Par exemple, une qualité estimée de $98 \%$ signifie que deux mots sur cent sont potentiellement erronés ${ }^{30}$. " Toutefois, on ne trouve pas de complément d'information sur ce «taux de reconnaissance estimé ". Plus encore, l'investigation des fichiers ALTO ${ }^{31}$ (disponibles via l'API de Gallica ${ }^{32}$ ) montre que, parmi les 1980 pages du corpus pour lesquelles une océrisation de la BNF existe, 1734 l'ont été par Isako $^{33}, 168$ par ABBYY ${ }^{34}$ tandis que 78 d'entre elles ne renseignent pas l'outil. Cette pluralité d'outils traduit donc une diversité de campagnes d'océrisation - comme le suggère justement l'attribut ID de la balise ocrProcessing, prévoyant ainsi, au sein même de la structure de données, la possibilité d'une succession de plusieurs campagnes d'OCR. Derrière le " taux de reconnaissance estimé " se cachent donc divers outils, différentes campagnes d'OCR, réalisées à différents moments, rendant peu significative, voire vaine, la comparaison de deux résultats d'OCR par cette mesure.

Ce constat justifie notre volonté d'océriser le corpus selon un protocole unique, utilisant un modèle libre et appris sur des données du $\mathrm{XVII}^{\mathrm{e}}$ siècle, et de l'évaluer par des métriques clairement exposées ${ }^{35}$.

\section{Influence du bruit et du silence sur les tables de fréquences}

L'acquisition des données textuelles par OCR, dans une démarche d'analyse des faits de langue et de style sur corpus, pose d'entrée de jeu les deux problèmes du bruit et du silence. Le premier, pluriel en tant que résultat d'une océrisation d'une portion de texte inexistante ${ }^{36}$ mais aussi en tant que confusion entre caractères " proches $^{37}$ ", a pour double effet d'ajouter de fausses entrées dans les tables de fréquences et de diminuer la fréquence des entrées qui ont été mal reconnues. Le second, en tant qu'absence de reconnaissance de portions de texte pourtant présentes dans le document numérisé, implique une diminution des fréquences des formes non reconnues - et certains hapax deviennent des «nullax ». L'analyse de ces tables de fréquences ne peut donc être réalisée que dans l'observation de phénomènes saillants. Autrement dit, cette étude sur corpus bruité ne peut rendre compte de la rareté des phénomènes : une des conclusions (négative) de cette étude est que la quête des néologismes ne peut pas être menée systématiquement avec une telle qualité de données - voir ci-dessous pour une approche plus fine par les rimes qui autorisent toutefois quelques conclusions sur ce point ${ }^{38}$. 


\section{Exploration contrastive des écrits burlesques de la Fronde}

La caractérisation des spécificités d'une langue ou d'un genre peut se faire au moyen de corpus contrastifs, ou corpus de référence (Rastier 2011, 33). L'établissement de tels ensembles doit répondre à des critères explicites, mais, lorsque tout est à construire, il faut parfois faire des compromis entre ce qui est possible et ce qui est désirable (Hunston 2008).

\section{Description des corpus contrastifs}

La délimitation de ce que sont les textes burlesques pose en soi un problème comme vu plus haut : sans redéfinir la notion, nous nous sommes fondés sur les 45 titres cités par Francis Bar (1960) dans Le Genre burlesque en France au XVII siècle. Étude de style ${ }^{39}$. Concernant l'obtention des données elles-mêmes, elle a été contrainte par la disponibilité numérique. Au-delà de l'arbitraire de ces choix, il s'agissait d'acquérir un corpus de référence d'une taille suffisante pour être statistiquement représentatif. S'il n'existe pas de taille idéale minimale, on considère souvent comme le fait Sinclair (2003) qu'un million de tokens pour chaque sous-corpus à comparer est une taille raisonnable.

Le caractère opportuniste (McEnery et Hardie 2011) de nos corpus induit un défaut de calibrage générique. Ainsi le théâtre y a-t-il sans doute une place plus importante en raison du corpus en ligne Théâtre classique, qui a constitué une partie de nos sources. Le projet e-ditiones ${ }^{40}$, qui rendra disponible en 2021 un corpus de textes du XVII ${ }^{\mathrm{e}}$ siècle présentant tous les genres de discours alors pratiqués (genres littéraires, droit, sciences, correspondances, etc.) pourrait permettre à l'avenir de pallier ces défauts de calibrage (Gabay, Clérice et Reul 2020). Par ailleurs, la qualité et l'enrichissement de ces diverses données sont hétérogènes, depuis la sortie brute d'OCR (notre corpus d'étude, BuRLESQue FronDE), jusqu'à des données lemmatisées, POS-taggées et rendues disponibles en TEI (Presto et Frantext), utilisées pour les corpus de contraste.

TABLEAU 1. DESCRIPTION SYNTHÉTIQUE DES CORPUS : NOMBRE D'ÆEUVRES, NOMBRE D'OCCURRENCES ET NOMBRE DE FORMES (CALCULÉES AVEC ANTCONC)

\begin{tabular}{|l|l|l|l|}
\hline Corpus & $\begin{array}{l}\text { Nombre } \\
\text { d'œuvres }\end{array}$ & $\begin{array}{l}\text { Nombre } \\
\text { d'occurrences }\end{array}$ & $\begin{array}{l}\text { Nombre de } \\
\text { formes }\end{array}$ \\
\hline NON BURLESQUE & 1092 & 7873298 & 231283 \\
\hline BURLESQUE & 235 & 3525879 & 256909 \\
\hline BURLESQUE FRONDE & 179 & 3123098 & 230889 \\
\hline $\begin{array}{l}\text { BURLESQUE HORS } \\
\text { FRONDE }\end{array}$ & 56 & 402781 & 50642 \\
\hline BURLESQUE VERS & 202 & 1733044 & 170243 \\
\hline BURLESQUE PROSE & 33 & 1792835 & 134855 \\
\hline
\end{tabular}


56 œuvres dites "burlesques " par la tradition ont été rassemblées pour constituer un corpus burlesque contrastif (hors Fronde, donc). C'est donc à partir de la bibliographie de Bar (1960) que nous avons cherché les textes libres de droits dans le sous-ensemble «1500-1700 » du corpus Frantext ${ }^{41}$ puis sous forme de PDF dans les bibliothèques numériques en ligne (nécessitant océrisation en ce cas).

Parallèlement, 1092 œuvres non burlesques ont été rassemblées pour constituer un second corpus contrastif ; il s'agit de l'agrégation des œuvres repérées comme non burlesques figurant dans les corpus Frantext, SERMO ${ }^{42}$ et Théâtre classique ${ }^{43}$. La volumétrie des différents corpus utilisés dans notre étude est présentée dans le tableau $1^{44}$. On y voit aussi la partition vers/prose que nous avons jugé bon d'effectuer.

\section{AntConc}

Nous avons choisi de réaliser des expériences avec l'outil AntConc pour montrer que l'exploitation d'un corpus bruité ne nécessitait pas d'outillage complexe ni de préparation intensive des données. Cet outil présente l'avantage de poser peu de contrainte sur le format des données d'entrée, d'être multiplateformes et de fonctionner sans installation préalable, permettant ainsi de juger rapidement de l'utilisabilité d'un corpus. Par ailleurs, il exploite des mesures statistiques bien connues et validées par la communauté scientifique comme l'information mutuelle (Kullback 1959) pour mesurer les collocations (onglet collocates). Pour ce qui est de l'utilisation de corpus contrastifs (reference corpus dans AntConc), il permet de vérifier la spécificité d'un terme (keyness) au moyen de la log-likelihood (Fischer 1922) ou du $\chi^{2}$ (Chernoff et Lehmann 1954). Nous avons choisi la mesure log-likelihood (4 terms), mesure par défaut dans AntConc, avec une valeur $p<0,05^{45}$. Plus la valeur de la mesure loglikelihood est élevée pour un terme, plus celui-ci est spécifique du corpus étudié.

Bien entendu, se pose la question de la qualité des observables que l'on peut tirer d'un tel corpus bruité mais les patrons de tokenisation utilisés par AntConc ne semblent pas affectés outre mesure par le bruitage de l'entrée ${ }^{46}$. On peut le prouver par un test simple consistant à montrer la spécificité des lexies référant à l'actualité : le contraste entre les écrits burlesques produits pendant la Fronde et ceux produits avant ou après (Burlesque Fronde $v s$. Burlesque hors Fronde) montre une fréquence supérieure de noms propres dans notre corpus d'étude. Apparaissent ainsi en haute fréquence les principaux anthroponymes attendus (ceux des personnalités impliquées dans les événements : Mazarin, Condé, Beaufort) ou des partis en présence (Parlement) ainsi que les appellatifs ou les désignateurs associés : Messieurs (désignant les parlementaires) ou partisans (pour les financiers enrichis et associés à Mazarin). Le résultat de ce test ne prouve rien de la spécificité des textes burlesques d'actualité (la spécificité des noms propres aurait certainement été moins saillante avec un corpus contrastif d'imprimés dits "occasionnels ", imprimés eux aussi en lien avec un fait d'actualité) : il sert à montrer que le bruit (dans les données) peut certes produire du silence (dans les résultats), mais que cela ne gêne que marginalement le repérage des unités lexicales saillantes. 


\section{Usage polémique du motif de la " muse burlesque "}

Un premier cas de spécificité peut être étudié avec le mot " muse ». Il apparaît dans les hautes fréquences du corpus BURLEsQue FrondE, au rang 520 sur les 50642 formes du corpus, ce qui représente 63 occurrences, et 77 en incluant la flexion du mot au pluriel. Le mot apparaît également dans les spécificités positives du corpus BURLESQuE face au corpus Non BURLESQUE, sans que ce résultat soit original, puisque le motif de la muse est très fréquent en poésie (il l'est donc dans le corpus BURLESQue, majoritairement poétique) alors que le corpus NoN BURLESQUE mélange prose et poésie. C'est l'usage spécifique de ce motif qui nous intéressera.

Les indices énonciatifs les plus associés au mot dans les écrits burlesques (repérés grâce à la fonction Clusters/ $\mathrm{N}$-grams d’AntConc) indiquent une invocation récurrente à cette allégorie de l'inspiration, grâce aux marques de l'interlocution : " ma muze ", " ma muse ", " chere mufe ", " notre muse ", " toy mufe "; le « o " apparaît également dans les cooccurrents fréquents, signalant un emploi "invocatif " (Grinshpun 2006). L'invocation à une muse "burlesque " ou " grotesque " a déjà été repérée comme typique du burlesque dès avant la Fronde (Nédélec 2020, 432-433). La fréquente invocation à la " muse " est attendue dans un corpus poétique du $\mathrm{XVII}^{\mathrm{e}}$ siècle, le motif étant peut-être celui qui est le plus repris en poésie depuis le siècle précédent ${ }^{47}$, en particulier d'une manière parodique (antiépique et anticlassique) ou transgressive, notamment chez les satiriques (Debailly 2012). Depuis lors convoquées souvent pour être récusées, les muses cristallisent une attitude métapoétique qui fait par ailleurs partie des caractéristiques de l'écriture burlesque (Cronk 1987). L'invocation-révocation de la muse n'est pas ainsi essentiellement la marque du travestissement épique - qui a pu être vu un peu trop rapidement comme le «style burlesque le plus typique de la Fronde " (Carrier 1996) -, car ce motif de l'invocation à la muse n'indique pas une réécriture triviale du modèle épique, mais se réduit à " une vague allusion » et à un « emprunt isolé » (Nédélec 2004). De fait, on ne trouve pas dans les corpus burlesques fouillés ici d'autres mots en hautes fréquences qui renverraient à la mythologie, par contraste avec les autres textes de l'époque (corpus NoN BURLESQUE), où la référence antique est très vivace.

Les adjectifs caractérisants que fait apparaître la recherche de cooccurrences sont les suivants : "marmote " (au sens de "grotesque "), " boufonne ", "falote " (au sens d' "impertinent " et de " drôle »), " grotesque ", " badine ». On vérifie ainsi que ces écrits des années 1648-1653 poursuivent la déconstruction de la figure mythologique de l'inspiration : sans doute cette allégorie est-elle un symbole voyant qu'il est efficace de déboulonner, en disqualifiant les antiques muses comme "vieilles et camuses ", en renvoyant dos à dos merveilleux païen et chrétien (Nédélec 2020) et en faisant par plaisanterie de leurs avatars des réalités triviales (dans le corpus d'étude, " pégaze " rime avec " aze ", c'est-à-dire « âne »).

D'autres cooccurrents comme " rire », "petite » ou " pauvre " pointent vers cette démystification de la figure du poète que l'écriture burlesque met en jeu : non plus d'origine transcendante, la poésie prend désormais sa source dans l'expérience du poète, ou une vision " humorale ", où la 
muse se voit remplacée par l'humeur, ou la verve (Folliard 2020). Le mot " humeur » est d'ailleurs fréquemment présent ( $61^{\mathrm{e}}$ rang de fréquence), notamment dans un sens métapoétique, comme en attestent les cooccurrents adjectivaux postposés : " folle ", " badaude ", " crotesque ", " debonnaire ", " insolente ", " mutine ", " plaisante ", " soldatesque "; les $n$ grammes les plus fréquents à gauche permettent d'accéder aux caractérisants adjectivaux antéposés : «bonne ", " gaillarde » ou «belle »; l'expression «belle humeur » est en effet quasi synonyme de "burlesque " à l'époque, comme en témoigne d'Assoucy (1650).

L'allusion à une muse triviale fonctionnerait ainsi comme un signal stylistique d'une pratique burlesque autonomisée et qui se commente elle-même, plus que comme déclencheur d'une parodie de l'épique ou comme révocation transgressive de l'antique. La position du mot dans l'économie textuelle de certains écrits peut conforter cette intuition. On peut ainsi observer synthétiquement la présence du mot " muse " dans chacun des textes grâce à une fonctionnalité du logiciel AntConc nommée concordance plot, qui permet de spatialiser schématiquement les zones d'emploi d'un mot dans chaque texte (voir figure 5).

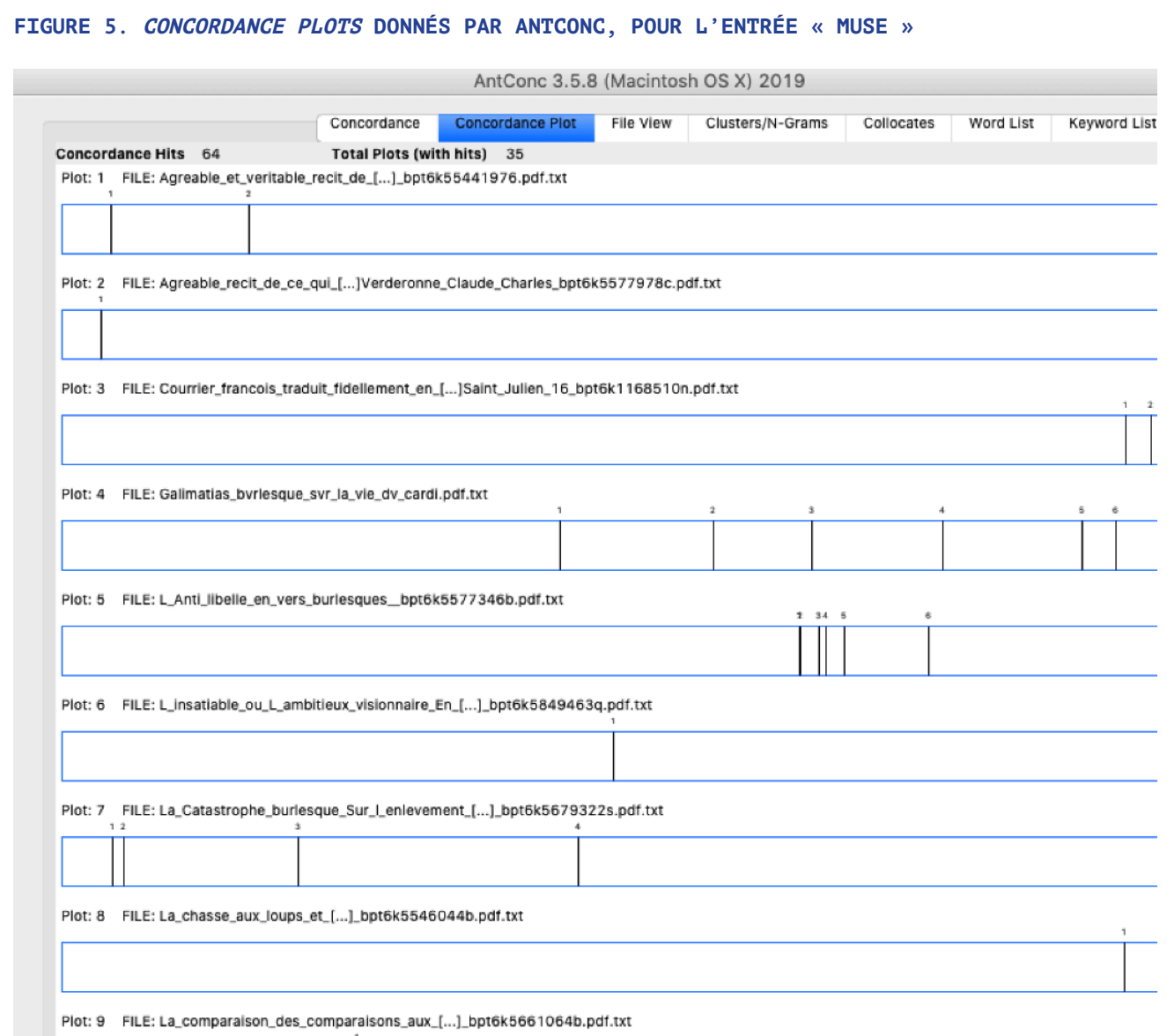

Le mot n’apparaît ainsi pas seulement au début des pièces, ce qui suggère qu'il n'est pas cantonné à un pur emploi invocatif. Cette visualisation nous indique par exemple une forte fréquence du mot à la fin de certains écrits ; on peut s'arrêter sur L'antilibelle en vers burlesques (Paris, Pierre du Pont, 1649), p. 9 :

N'en doutez point efprit de crotte, En qui quelque mufe marmote, Mufe efprit de rebellion [...] Mufe fourde borgne \& boiteufe Qui porte des haillons de gueufe, 
Mufe au nez fans cefle morueux

A qui chaftie outre les yeux, [...]

Mufe dont les cheueux en tefte,

Sont befte d'vne pire befte [...]

Car cette noire milufine

Eft fuiette de proferpine,

Elle eft engendrée en Enfer,

Son grand pere c'eft Lucifer

Et n'eftoit montée fur terre,

que pour y fomenter la guerre,

C'eft là bourruë \& fols efprits

Le genie de vos efcrits [...]

On observe une délocution de l'objet « muse ", qui n'est plus l'allégorie à qui on s'adresse, mais bien une caractéristique des poètes adverses, qui devient thème de l'énoncé et dont il s'agit de prédiquer des propriétés négatives. Cet écrit imprimé après la paix de Saint-Germain en avril 1649 apparaît comme une réponse au flot de pièces burlesques parues pendant les trois mois qui ont précédé (le siège de Paris, qui a suscité au moins 500 écrits ${ }^{48}$, dont de nombreux en vers burlesques). Le mot "muse » est ici non plus allégorique mais métonymique, utilisé pour viser collectivement les poètes ayant publié pendant le blocus pour le dénoncer : le stylème pseudo-épique est récupéré dans un sens polémique.

\section{Le « burlesque On "}

Les spécificités lexicales obtenues en opposant le corpus BuRLESQue FRONDE au corpus BURLESQUE HORS FRONDE montrent une surreprésentation des indéfinis, en particulier du pronom personnel indéfini « on »d'ailleurs non sujet à la flexion ni à la variation orthographique, ce qui rend ce résultat d'autant plus fiable. Dans notre corpus d'étude, on recense 3452 occurrences de ce pronom, utilisé avec marquage stylistique dans deux types d'emploi : la personnification et l'anaphore.

TABLEAU 2. SPÉCIFICITÉ DU « ON » AVEC DIFFÉRENTES CONFIGURATIONS DE CONTRASTE

\begin{tabular}{|l|l|l|}
\hline Corpus d'étude & Corpus de référence & Spécificité \\
\hline BURLESQUE & NON BURLESQUE & 822 \\
\hline BURLESQUE HORS FrONDE & NON BURLESQUE & 370 \\
\hline BURLESQUE FroNDE & BURLESQUE HORS FRONDE & 333 \\
\hline BURLESQUE FRONDE & BURLESQUE + NON BURLESQUE & 571 \\
\hline
\end{tabular}




\section{Propagation et personnification de la rumeur}

Ce pronom apparaît largement dans des énoncés invoquant ou propageant une rumeur. L'observation simple du concordancier couplée à un tri des occurrences (selon leur contexte droit ou gauche) permet de compter un nombre important d'occurrences associées au verbe de discours rapporté " dire " : " on dit que ", " qu'on dit ", " comme on dit ", " on m'a dit ", " comme l'on dit ", etc. Notons aussi les incises " (dit-on) » et « (ce dit-on) » qui utilisent les parenthèses, soulignant par la typographie le désengagement énonciatif du scripteur, et la plaisante mise en scène de celui-ci. Ces imprimés éphémères de la Fronde ont ainsi diffusé à grande échelle un des procédés typiques des écrits d'actualité depuis la fin du Xvi ${ }^{\mathrm{e}}$ siècle : colporter, inventer, mettre en scène la rumeur.

Outre ces verbes introducteurs de discours rapportés, le tableau de fréquences permet de relever des figures, notamment la personnification de la rumeur, par nominalisation du pronom. D'origine étymologique ( « on » vient de " homme »), cette figure est loin d'être une création burlesque : figée en catachrèse, elle est alors proverbiale, comme l'atteste le Dictionnaire de Furetière, où sujet et verbe sont nominalisés ensemble dans le proverbe : "On dit est un grand menteur, pour dire, que les bruits du commun sont souvent faux ». Mais la récurrence et la variété des emplois montrent comment ces imprimés exploitent toutes les virtualités d'une phraséologie. Il existe par exemple un périodique (à la brève existence, ayant connu 6 livraisons) titré Le Burlesque On de ce temps (avec variantes). Nous en avons trouvé 3 numéros disponibles en ligne en $\mathrm{PDF}^{49}$. La figure y est attestée (par les moyens qui sont les nôtres) 6 fois (sous la forme « cet $\mathrm{ON}$ » et variantes graphiques ${ }^{50}$ ) et 1 fois dans le syntagme " mon $\mathrm{ON}$ " dans Le Qu'en dira-t-on de Mazarin, burlesques (Paris, Antoine Quenet, 1649). L'usage figuré reste donc relativement exceptionnel, puisque dans les quatre pièces sus-citées, il est attesté 7 fois, contre 208 occurrences d'emploi standard du pronom.

Mais les fonctionnalités d'AntConc, indifférentes à la casse, permettent de repérer des dispositifs graphiques intéressants : on remarque en effet l'usage des capitales, destiné à rendre plus visible le mot, et sans doute la figure ${ }^{51}$ : dans Le Burlesque On de ce temps renouvelé, qui sait, qui fait et qui dit tout ce qui s'est passé depuis la guerre. Première partie, on trouve 90 occurrences du « $\mathrm{ON}$ » en majuscules. Cet artifice typographique montre comment ces écrits filent une figure banale jusqu’à en exploiter toutes les ressources, y compris syntaxiques (le pronom acquiert les caractéristiques d'un nom, voire d'un nom propre, puisque l'initiale est souvent capitalisée) et visuelles ${ }^{52}$. 
L'observation des concordance plots permet de révéler les passages du corpus où le " on » est surreprésenté (disons plutôt, concentré). Les anaphores en "on " apparaissent récurrentes, le pronom étant sujet de verbes dénotant des procès, du type « on court, on s'avance, l'on fuit ${ }^{53}$ ". On compte 29 mazarinades présentant ce type d'énumérations ${ }^{54}$. Ce stylème a plusieurs effets. D'abord, il peut tendre à mettre sur le même plan plusieurs groupes différents, troublant la compréhension claire du schéma actantiel ; par suite, il participe d'une écriture figurative, en particulier pour évoquer des personnages non individués et des mouvements de foule. Il s'agit d'un emploi de " on " qu'on pourrait dire " de multitude ", qu'on retrouverait dans la satire. C'est ce que montre l'extrait de l'Agréable récit de ce qui s'est passé aux dernières barricades de Paris, décrites en vers burlesques (de Verderonne, N. Bessin, 1649, p. 10) :
Le peuple fait les Barricades;
Les pourfuiuant auec brauades
De tous coftez on fait grand bruit,
On court, on s'auance, l'on fuit,
Maçons, Charpentiers, Eftuuiftes
Imprimeurs, Relieurs, Copiftes, [...]

On voit comment le repérage d'une simple unité lexicale comme le " on " peut embrayer sur le repérage d'autres stylèmes, comme l'énumération, voire l'accumulation de noms (la liste des noms de métiers a été ici abrégée, mais elle se poursuit sur 19 vers). On repère ainsi un outil d'amplification caractéristique, qui occasionne les innovations poétiques typiques du burlesque (ici l'usage de technolectes en poésie).

Ensuite, la caractéristique de " on " de pouvoir renvoyer à tout sujet animé indéfini permet de flouter les références et de servir la dénonciation. Le flou dans la référence des pronoms avait déjà été noté par (Jouhaud 2009, 109), comme une manière de chercher l'adhésion du plus grand nombre en maintenant l'ambiguïté ou d'insinuer des assertions mensongères. Ainsi, l'extrait suivant, tiré de l'Agréable et véritable récit de ce qui s'est passé devant et depuis l'enlèvement du roi hors de la ville de Paris par le conseil de Jules Mazarin, en vers burlesques, Paris, J. Guillery (p. 10), vise à insister sur le rejet unanime de Mazarin, pour mieux rendre scandaleuse sa carrière sans entraves (évoquée après l'adversatif « mais ») :

On commence de le cognoistre,

On s'appercoit que c'eft vn traiftre,

Que pour attraper le tefton,

Il pippe comme vn beau demon.

On quitte là fa compagnie,

On le fuit comme vne Harpye,

On le laiffe comme vn trompeur,

Où pluftoft comme vn fin voleur,

Contre luy on crie, on murmure,

Mais le pis, il va fe mocquant,

Car il a gaigné leur argent,

Le repérage de ces deux dernières séquences permet de voir l'orientation argumentative divergente des écrits présentant les mêmes stylèmes, le premier (l'Agréable récit) évoquant avec une certaine morgue les émeutes du "peuple ${ }^{55}$ ", alors que le second (l'Agréable et véritable ré- 
cit) est clairement hostile à Mazarin. La proximité des titres suggère d'ailleurs que le second répond au premier, reprenant des tours d'écriture en un sens argumentatif différent.

Ces deux exemples (allégorie de la " muse burlesque ", devenue métonymie des poètes productifs pendant le siège de Paris début 1649, et emploi énumératif ou pamphlétaire du « on » de multitude) montrent les atouts du repérage statistique de spécificités contrastives, surtout s’il est combiné à des fonctionnalités comme la visualisation de la textualité, l'examen des cooccurrents et le tri des occurrences par casses.

\section{Des rimes typiques du burlesque?}

Puisque le corpus présente une véritable homogénéité de genre (poésies étiquetées comme vers burlesques à $97 \%$ ), nous examinerons les rimes, de manière interne.

La première expérience consiste à relever les $n$-grammes de caractères les plus fréquents en fin de ligne. Cette première approche des rimes peut mettre au jour des rimèmes (Beaudouin 2000) typiquement burlesques. Pour juger de la fréquence des rimes dans nos données non enrichies, on doit combiner les formes fléchies des finales (singulier et pluriel notamment). Par exemple la rime en "-ade » apparaît 275 fois, ce qui fait partie des hautes fréquences : "bravades ", " rodomontades ", " barricades ", " estocades ", " embuscades ", " capilotades ", " algarades ", " boutade ", " mascarade ", " camarade ", " mousquetade ", " saluades ", " gourmades " (" coups de poing "), " grenade " (au sens de " petite bombe »), " incartades ", " rebuffades ", " fanfaronnades ", " mascarades ", "salade "- au sens technique de " casque ", souvent réservé à l'évocation de guerres antiques selon le Dictionnaire de l'Académie (1694). Sur le plan lexical, l'ensemble exemplifie bien le mélange sociolectal, avec des termes techniques ("capilotades ", " salade ") à côté de mots à connotation familière ou péjorative, souvent associés dans une isotopie de la guerre décrite comme une "bastonade ». Le sémantisme du suffixe "-ade " (puisqu'en l'occurrence la rime est un morphème dérivationnel, les mots-rimes étant essentiellement des noms) renvoie en outre à l'idée d'action ou de collectif, d'après le Trésor de la langue française ${ }^{56}$. Les titres d'œuvres néologiques (Juliade, Miliade et Mazarinade) exploitent cette polysémie du suffixe : à la fois formateur de titres d'œuvres épiques (le sens collectif renvoyant alors au grand nombre de parties composant de telles œuvres) et vecteur de connotations péjoratives.

Cet élément d'explication générique (le burlesque comme parodie d'épopée) n'est néanmoins pas le seul : un dictionnaire de rimes de l'époque, particulièrement sensible aux italianismes (La Noue 1623) cite les rimes en "-ade » comme presque toutes issues de noms italiens en « ata ", tout en précisant qu'" on en pourra adjouter encore icy de nouveaux [...] quand la pratique les aura un peu adoucis ». Ces mots-rimes semblent donc, d'une part être encore sentis comme des emprunts (conformément à la tendance antipuriste du burlesque) et, d'autre part apparaître comme relativement rares puisque La Noue indique que sa liste pourrait être augmentée à mesure que l'usage acclimaterait ces mots italiens au français. On approche ici finalement l'hypothèse de la poésie burlesque de la Fronde comme productive en néologismes. Le 
mot "frondade " en fournit un exemple : " Dès les premieres barricades / sans recommencer les frondades " (Tableau raccourci des courtisans, 1649, p. 3, emprunté à une chanson de Scarron).

Enfin, une recherche de la spécificité de cette rime peut être menée par une confrontation avec le rimarium de Beaudouin (2000). Les rimèmes en "-ade(s) » y sont au nombre de 26 occurrences (sur 80 ooo vers étudiés), avec 19 paires de mots-rimes différentes, soit parmi les plus rares de ce corpus. Aussi, cette association de phonèmes pourrait sonner comme spécifique du burlesque et donnerait à cette pratique d'écriture et de lecture sa couleur et sa voix - car ces écrits étaient vraisemblablement souvent oralisés (par des lecteurs aguerris à destination de moins lettrés, par des colporteurs, voire par des performances chantées pour certains écrits).

Le même travail pourrait être mené sur des rimes qui semblent intuitivement rares ailleurs et qui sont fréquentes dans le corpus d'étude, comme la rime en "-ique » (qui apparaît au $10^{\mathrm{e}}$ rang du calcul de spécificité du corpus BURLESQUE FrONDE) ou la rime en " -if ${ }^{57}$ ". Il faudrait pour affermir ces constats bénéficier d'un corpus de référence spécifiquement poétique et finement partitionné par genres (sur l'importance du genre pour la constitution des corpus de référence, voir Rastier 2011).

\section{De l'intérêt des données imparfaites et des moyens raisonnés de leur standardisation}

Ces premières explorations permettent d'assurer l'intérêt de notre hypothèse inaugurale (la fécondité des données bruitées utilisées en corpus) en prouvant leur exploitabilité (existence de phénomènes fréquents) et en dessinant certaines limites (dont notamment l'étude réduite de la rareté). Toujours est-il que, même si elles nous privent de certaines voies d'étude, les données océrisées - et donc imparfaites au sens où elles sont « bruitées ${ }^{58}$ »- permettent l'étude textométrique. En outre, le processus d'OCR (qu'on cherche à maîtriser par ses évaluations) contrecarre la nécessaire transcription manuelle, longue et coûteuse ; on dispose donc virtuellement d'une quantité supérieure de textes propices à l'étude.

Des questions restent bien sûr en suspens : le "bruit " des données océrisées relève-t-il plus précisément du bruit ou du silence ? En clair, les données océrisées contiennent-elles (i) de faux éléments ajoutés ou (ii) de véritables éléments ôtés ? Par exemple, les termes obscènes, qu'on associe généralement aux écrits burlesques, n’apparaissent pas dans les spécificités calculées entre le corpus BURLESQUE et le corpus NoN BURLESQUE ; faut-il y voir la conséquence d'un silence trop important dans les données ? Ou une confirmation de Nédélec (2004) qui mettait en doute le fait que l'écriture burlesque soit la seule caractérisée par un tel lexique?

Au demeurant, plusieurs tâches en cours visent à améliorer la qualité des données et des métadonnées sans forcément passer par une transcription de l'ensemble des quelque 5 ooo écrits recensés. D'abord, une transcription manuelle (ou obtention d'une vérité de terrain) d'au moins quelques échantillons est en cours, qui entraîne le modèle d'OCR et l'adapte aux données concernées (fine tuning) ${ }^{59}$. Elle permettra d'amélio- 
rer les performances du modèle ici utilisé (Gabay, Clérice et Reul 2020) et d'obtenir des données plus lisibles et partageables avec la communauté. Ensuite, une structuration dans un format XML-TEI élémentaire est également en cours d'établissement nonobstant le bruitage du corps du texte ; cette tâche est semi-automatisée par des scripts convertissant automatiquement les sorties d'OCR et ajoutant des balises minimales (paragraphes, sauts de pages et de ligne, et header corrigé avec récupération manuelle des données de catalogage et des bibliographies récentes sur le sujet ${ }^{60}$ ). Enfin, le travail sur les métadonnées est une tâche actuellement menée de manière complémentaire et indépendante du travail sur les données elles-mêmes, en vue d'une exploitation immédiate par la communauté ${ }^{61}$.

\section{Bibliographie}

Académie française. 1694. Le Dictionnaire de l'Académie françoise. Paris : $\mathrm{V}^{\mathrm{ve}}$ Jean-Baptiste Coignard.

Assoucy, Charles Coypeau d'. 1650. L'Ovide en belle humeur de Mr Dassoucy. Enrichy de toutes ses figures burlesques. Paris : Charles de Sercy.

Bar, Francis. 1960. Le Genre burlesque en France au XVII ${ }^{e}$ siècle. Étude de style. Paris : Éditions D’Artrey.

Beaudouin, Valérie. 200o. « Rythme et rime de l'alexandrin classique. Étude empirique des 80 ooo vers du théâtre de Corneille et Racine ". Thèse de doctorat en sciences du langage, École des hautes études en sciences sociales. https://tel.archives-ouvertes.fr/tel00377348.

Briot, Frédéric. 1993. "La rime et la déraison : l'épître en vers dans le temps de la Fronde ". Littératures classiques 18 : 159-171. https://doi.org/10.3406/licla.1993.1721.

Burgy, Florence, Steeve Gerson et Loïc Schüpbach. 2020. "Ex imagine ad litteras : projet d'océrisation de la collection De Bry ". Mémoire de master en sciences de l'information, Haute école de gestion de Genève.

Carrier, Hubert. 1989. La Presse de la Fronde (1648-1653) : les mazarinades. La Conquête de l'opinion. Genève : Droz.

Carrier, Hubert. 1996. Les Muses guerrières. Paris : Klincksieck.

Chernoff, Herman, et E. L. Lehmann. 1954. «The Use of Maximum Likelihood Estimates in $\chi^{2}$ Tests for Goodness of Fit ". Annals of Mathematical Statistics 25 (3) : 579-586.

Cronk, Nicholas. 1987. «La défense du dialogisme : vers une poétique du burlesque ». Dans Burlesque et formes parodiques. Actes du colloque de l'université du Maine, Le Mans, 4-7 décembre 1986, édité par Isabelle Landy-Houillon et Maurice Ménard, 321-338. Seattle : Papers on French Seventeenth Century Literature.

Dandrey, Patrick, éd. 1996. Dictionnaire des lettres françaises. Publié sous la direction du Cardinal Georges Grente. Édition entièrement révisée, amendée et mise à jour. Paris : Fayard.

Debailly, Pascal. 2012. La Muse indignée. t. 1 : La Satire en France au Xvie siècle. Paris : Classiques Garnier.

Fischer, Ronald Aymler. 1922. "On the Mathematical Foundations of Theoretical Statistics ". Philosophical Transactions of the Royal Society of London Series A 222 : 309-368. http s://doi.org/10.1098/rsta.1922.0009.

Folliard, Melaine. 2020. «Le bannissement des Muses : Théophile de Viau ou l'immanence de l'inspiration ". Littératures classiques 102 (2) : 47-61. https://doi.org/10.3917/licla1.102.0047.

Furetière, Antoine. 1690. Dictionnaire universel contenant généralement tous les mots français tant vieux que modernes. La Haye et Rotterdam : A. et R. Leers.

Gabay, Simon, Thibault Clérice et Christian Reul. 2020. "OCR17 : Ground Truth and Models for 17th c. French Prints (and Hopefully More) ». Document de travail. https://hal.archives-ouvertes.fr/hal-02577236. 
Galand-Hallyn, Perrine et Anne-Pascale Pouey-Mounou, éd. 2016. La muse s'amuse. Figures insolites de la muse à la Renaissance. Genève : Droz.

Genette, Gérard. 1982. Palimpsestes. La Littérature au second degré. Paris : Le Seuil. http://b anq.pretnumerique.ca/accueil/isbn/9782021184006.

Grinshpun, Yana. 2006. «Fait de langue et fait de style : Ô dans les Élégies de Chénier et dans Tête d'or de Claudel ». L'Information grammaticale 108 (1) : 27-31. https://doi.org/10.34 06/igram.2006.3798.

Gupta, Anshul, Ricardo Gutierrez-Osuna, Matthew Christy, Boris Capitanu, Loretta Auvil, Liz Grumbach, Richard Furuta et Laura Mandell. 2015. " Automatic Assessment of OCR Quality in Historical Documents ». Dans Proceedings of the Twenty-Ninth AAAI Conference on Artificial Intelligence, 1735-1741. Palo Alto : AAAI Press. https://ojs.aaai.org/index.php/AAAI/article/view/9487.

Hill, Mark J., et Simon Hengchen. 2019. « Quantifying the Impact of Dirty OCR on Historical Text Analysis : Eighteenth Century Collections Online as a Case Study ». Digital Scholarship in the Humanities 34 (4) : 825-843. https://doi.org/10.1093/llc/fqz024.

Hunston, Susan. 2008. «Collection Strategies and Design Decision». Dans Corpus Linguistics. International Handbook, édité par Anke Lüdeling et Merja Kytö, 154-167. Berlin : De Gruyter.

Jiang, Ming, Yuerong Hu, Glen Worthey, Ryan C. Dubnicek, Boris Capitanu, Deren Kudeki et J. Stephen Downie. 2021. « The Gutenberg-HathiTrust Parallel Corpus : A Real-World Dataset for Noise Investigation in Uncorrected OCR Texts ». Poster présenté à iConference 2021, 17-31 March. http://hdl.handle.net/2142/109695.

Jouhaud, Christian. 2009 [1985]. Mazarinades. La Fronde des mots. Paris : Aubier.

Kiessling, Benjamin. 2019. « Kraken : an Universal Text Recognizer for the Humanities ». Communication présentée à Digital Humanities Conference 2019-DH2019, Utrecht, Netherlands, 9-12 July.

Kullback, Solomon. 1959. Information Theory and Statistics. New York : Wiley.

La Noue, Odet de. 1623. Le grand dictionnaire des rimes françoises selon l'ordre alphabétique. Genève : M. Berjon.

Labadie, Ernest. 1904. Nouveaux suppléments à la bibliographie des mazarinades. Paris : Henri Leclerc.

Leclerc, Jean. 2013. « La faim des Parisiens en vers burlesques : sur quelques mazarinades du blocus (hiver 1649) ». Dans Les Histoires de Paris (XVI ${ }^{e}$-XVIII $e^{e}$ siècle), édité par Thierry Belleguic et Laurent Turcot, t. 2, 199-218. Paris : Hermann.

Maingueneau, Dominique. 1983. Sémantique de la polémique. Discours religieux et ruptures idéologiques au XVII ${ }^{e}$ siècle. Lausanne : LÂAge d'Homme.

McEnery, Tony, et Andrew Hardie. 2011. Corpus Linguistics : Method, Theory and Practice. Cambridge : Cambridge University Press.

Ménage, Gilles. 1650. Les Origines de la langue française. Paris : A. Courbé.

Moreau, Célestin. 1850-1851. Bibliographie des mazarinades. 3 vol. Paris : Jules Renouard.

Moreau, Célestin. 1862. "Supplément à la bibliographie des mazarinades ». Bulletin du bibliophile et du bibliothécaire, 786-829.

Moreau, Célestin. 1869. « Supplément à la bibliographie des mazarinades ». Bulletin du bibliophile et du bibliothécaire, 61-81.

Nédélec, Claudine. 2004. Les États et empires du burlesque. Paris : Honoré Champion.

Nédélec, Claudine. 2020. «Les Muses camuses des burlesques». Littératures classiques 102 (2) : 81-92. https://doi.org/10.3917/licla1.102.0081.

Novakova, Iva, et Dirk Siepmann. 2020. Phraseology and Style in Subgenres of the Novel. A Synthesis of Corpus and Literary Perspectives. Cham : Palgrave Macmillan. https://doi.org/1 0.1007/978-3-030-23744-8.

Peureux, Guillaume. 2007. Le Burlesque. Paris : Gallimard.

Pincemin, Bénédicte. 1999. "Construire et utiliser un corpus : le point de vue d'une sémantique textuelle interprétative ». Dans Atelier Corpus et TAL : pour une réflexion méthodologique. Actes de la conférence TALN, Cargèse, 12-17 juillet, édité par Anne Condamines, Marie-Paule Pery-Woodley et Cécile Fabre, 26-36, Cargèse : TALN.

Rastier, François. 2011. La Mesure et le Grain. Sémantique de corpus. Paris : Champion. 
Sinclair, John. 2003. " Corpora for Lexicography ». Dans A Practical Guide to Lexicography, édité par Piet van Sterkenburg, 167-178. Amsterdam : John Benjamins. https://doi.org/10.1 075/tlrp.6.21sin.

Socard, Émile. 1876. Supplément à la bibliographie des mazarinades. Paris : H. Menu.

Souvay, Gilles, et Jean-Marie Pierrel. 2009. "LGeRM : lemmatisation des mots en moyen français ». Traitement automatique des langues 50 (2) : 21-44.

Springmann, Uwe, Florian Fink et Klaus U. Schulz. 2016. « Automatic Quality Evaluation and (Semi-) Automatic Improvement of OCR Models for Historical Printings ». arXiv preprint. https://arxiv.org/pdf/1606.05157.pdf.

Tanguy, Jean-Baptiste. 2020. « Exploiter des modèles de langue pour évaluer des sorties de logiciels d'OCR pour des documents français du XVII ${ }^{\mathrm{e}}$ siècle ». Dans Actes de la $\sigma^{e}$ conférence conjointe JEP, TALN, RECITAL. Vol. 3 : Rencontre des étudiants chercheurs en informatique pour le TAL, édité par Christophe Benzitoun, Chloé Braud, Laurine Huber, David Langlois, Slim Ouni, Sylvain Pogodalla et Stéphane Schneider, 205-217. Nancy : ATALA.

Van Der Haeghen, Philippe. 1859. « Notes biographiques sur les mazarinades ». Bulletin du bibliophile belge 15 : 384-395.

\section{Notes}

1 «Burlesque » qualifiant tantôt style, registre, ou " genre d'écrire », comme on disait à l'époque qui nous intéresse (Ménage 1650). Sur le débat à propos de la définition "du " burlesque comme style, registre ou genre, voir Nédélec 2004, 269.

2 Des campagnes de dépouillement collectif et de lecture ont été menées, sur un maximum d'une centaine de libelles burlesques (Leclerc 2013) ; telle étude sur les épîtres en vers de Scarron et Saint-Amant laisse les œuvres produites par ces auteurs pendant la Fronde « délibérément de côté » (Briot 1993, 160).

3 Du moins dans sa version jugée au $\mathrm{XIX}^{\mathrm{e}}$ siècle, voire au $\mathrm{Xx}^{\mathrm{e}}$ siècle, obscène ou dégradée : le Trésor de la langue française cite par exemple Sainte-Beuve pour illustrer l'acception littéraire du mot «burlesque » : «Et le burlesque, autre fléau, le burlesque, cette lèpre des années de la Fronde [...]».

4 Le chiffre de 1300 écrits burlesques parus pendant la Fronde, soit un quart des mazarinades, a été avancé par Carrier (1989, s.v. " Mazarinades "), dans Dandrey (1996, 831) et dans Carrier $(1996,93)$ mais sans bibliographie associée.

5 À l'exception notable des recherches possibles sur le site http://mazarinades.org, proposant des requêtes lexicales sur une grande majorité de mazarinades parfaitement transcrites. Toutefois, on ne peut y confronter de corpus contrastif ni user de fonctionnalités statistiques avancées.

6 "À la classique dualité entre induction et déduction dans les disciplines d'observation, le renouvellement méthodologique favorisé par les corpus numériques engage à substituer le cycle suivant : (i) analyse de la tâche et production des hypothèses ; (ii) constitution d'une archive et sélection d'un corpus de référence ; (ii) constitution d'un corpus de travail ; (iii) traitement instrumenté de ces corpus, en contrastant corpus de travail et corpus de référence ; (iv) interprétation des résultats et retour aux sources pour valider l'interprétation. " (Rastier 2011, 13).

7 Reconnaissance optique de caractères (ou optical character recognition, contracté plus loin en OCR).

8 Mais aussi du silence puisque des formes peuvent disparaître.

9 Ayant connu des antécédents, notamment en Italie (Nédélec 2004, 153).

10 Nédélec $(2004,22)$ ajoute qu'elle implique un jeu, voire un renversement des «normes et des modèles, dans l'ordre de l'esthétique, comme dans l'ordre du linguistique, de l'éthique et de l'idéologie».

11 L'écriture burlesque caractérise soit des textes entiers, et ce de manière transgénérique (par exemple, pour Scarron, sa parodie d'épopée le Virgile travesti, son Roman comique, ou son libelle intitulé La Mazarinade), ou des portions de textes, irriguant nombre de productions du XvII ${ }^{\mathrm{e}}$ siècle. Mais la notion est transhistorique : voir Peureux 2007, 87-103. 
12 Bar (1960) consacre 300 pages sur 400 au lexique dans sa caractérisation du " genre burlesque " ; pour un autre point d'observation, en l'occurrence les figures, voir Nédélec 2004, 291-292.

13 Bibliographie des mazarinades de Célestin Moreau et ses suppléments successifs : Moreau (1850 ; 1862 ; 1869) ; Socard (1876); Labadie (1904); Van Der Haeghen (1859).

14 Ce nombre doit être revu à la hausse si l'on inclut, comme Célestin Moreau, des « textes fantômes " (dont on sait par les sources qu'ils ont existé mais dont on n'a pas conservé d'exemplaires), ainsi que les réponses ou les suites qui ont souvent été incluses par Moreau dans une seule et même entrée. Hubert Carrier $(1989,71)$ produit ainsi une estimation de 5200 écrits distincts (à $5 \%$ d'erreur près).

15 Voir http://kraken.re et https://pypi.org/project/kraken/.

16 Le résultat de l'océrisation n’a pas été parfaitement corrigé à la main. Voir le fichier Moreau_bib_mazarinades.ods : https:/github.com/Antonomaz/revue_HN_burlesque_fronde/. Pour une version interrogeable de ce tableur, voir http://antonomaz.huma-num.fr/tools/Biblio_Moreau.html (version avril 2021).

17 La refonte totale de la bibliographie des mazarinades est entreprise par la bibliothèque Mazarine depuis 2019 (1200 notices atteintes en janvier 2020, soit près de $20 \%$ de l'ensemble des données attendues à terme) : https://mazarinades.bibliotheque-mazarine.$\mathrm{fr} /$ pages/pr\%C3\%A9sentation/.

18 Dans deux poèmes qui ont toutes les apparences de textes de piété : La Passion de nostre Seigneur, en vers burlesques. Dediée aux Ames Dévotes, Paris, chez la veuve Jean Rémy, 1649 ; Extase de la France Mourant d'amour pour Jesus-Christ crucifié. En vers burlesques, Paris, Claude Morlot, 1649. Ces cas ont déjà été repérés comme des "faux " burlesques : voir Nédélec (2004, 344, 421, 442, 443) et Carrier (1996).

19 En prose (par exemple, la Lettre de remerciment envoyee au Cardinal Mazarin... avec la harangue de dame Denise), ou en vers (Le medecin politique, qui donne un souverain remede, pour guerir la France malade à l'extremité).

20 Ce qui amène à relativiser l'estimation de 1300 libelles en vers burlesques, le quart des mazarinades, selon Carrier (1996), qui n’a pas pu publier la liste des titres concernés. Certes, le mot «burlesque(s) " n'est pas toujours dans le titre, mais il l'est significativement dans la grande majorité des exemples cités par Carrier, et il serait étonnant qu'il ne le fût pas dans plus d'un millier de ces écrits. Les métadonnées rénovées que produit actuellement la bibliothèque Mazarine (voir note 17) intègrent les recherches menées par Carrier restées manuscrites, et pourront éclairer ce chiffre.

21 https://gallica.bnf.fr.

22 https://books.google.fr.

23 https://tinyurl.com/nappe-renversee-renard/.

24 https://github.com/e-ditiones/OCR17/.

25 Les fichiers texte sont disponibles dans le dossier « mazarinades_burlesques » de l'entrepôt https://github.com/Antonomaz/revue_HN_burlesque_fronde/.

26 Voir Burgy, Gerson et Schüpbach 2020 ; Jiang et al. 2021 ; Hill et Hengchen 2019 pour des exemples d'océrisation de documents historiques et l'étude de ce type de données textuelles océrisées et bruitées.

27 Les codes qui permettent ce calcul sur des sorties d'OCR en HTML sont donnés dans le dossier "programs_ocr_eval " de l'entrepôt https://github.com/Antonomaz/revue_HN_burlesque_fronde/.

28 Il existe en effet une notion de mot dans les sorties d'OCR, quoique très réductrice. Les formats enrichis comme les HTML et XML proposent des balises à l'échelle du mot, celuici étant uniquement le résultat de la segmentation par l'espace.

29 Les précautions sont de rigueur lors de l'usage des taux de confiance pour juger la qualité d'une sortie d'OCR : l'expérience montre qu'ils sont très souvent supérieurs à o,90.

30 Voir https://gallica.bnf.fr/edit/und/consulter-les-documents/, "Qualité de l'OCR ».

31 Analysed Layout and Text Object.

32 http://api.bnf.fr/api-document-de-gallica/.

33 Isako $@$ - Document Conversion System [DCS $]^{\circledR}$.

34 ABBYY FineReader Engine. 
35 Notons toutefois que la BNF a internalisé les campagnes d'océrisation de ses fonds documentaires depuis le mois de mars 2019, offrant ainsi une unité dans la procédure et dans l'évaluation.

36 Par exemple, la fausse reconnaissance de texte dans une illustration.

37 Par exemple, le « $\mathrm{G}$ » et le « $\mathrm{O}$ ».

38 D'autres biais non liés à la qualité des données mais à l'indistinction du texte et du péritexte doivent aussi être corrigés dans l'interprétation des tables de fréquences, comme la haute fréquence des noms propres, qui peuvent être répétés dans les titres courants ou les didascalies nominatives au théâtre.

39 On y trouve notamment Brebeuf, Chappelle, Colletet, Dassouci, Furetière, Loret, Ménage, Saint-Amant et Scarron, mais aussi des auteurs aussi divers que Bruscambille et Marivaux.

40 https://github.com/e-ditiones/.

41 https://www.frantext.fr.

42 http://sermo.unine.ch/SERMO/.

43 http://www.theatre-classique.fr/pages/programmes/PageEdition.php.

44 Le fichier «Corpus_metadata.ods ", fourni sur l'entrepôt https://github.com/jbtanguy/revue_HN_burlesque_fronde.git, renseigne l'ensemble des métadonnées des corpus précités.

45 La valeur $p$ (ou p value, en anglais) est un seuil statistique en dessous duquel le phénomène a peu de chances d'être dû au hasard.

46 Sachant que la tokenisation automatique n'est pas un problème résolu, que ce soit pour le français moderne ou le français préclassique.

47 Pour un bilan très complet, y compris bibliographique, sur ce point, voir Galand-Hallyn et Pouey-Mounou 2016.

48 Moreau en dénombre 621 dans son classement chronologique des mazarinades (Moreau 1850 , t. 3, 300-315).

49 Extraordinaire arrivée du burlesque On de ce temps qui sait, qui fait et qui dit toutes les particularités du siége de Cambray, avec un sommaire de l'ordre du festin fait aux généraux et Parlement d'Angleterre par les communes, 1649, chez E. Hébert (17 occurrences de « on »); Le buflesque On de ce temps renouvelé, qui sait, qui fait et qui dit tout ce qui s'est passé depuis la guerre. Première partie, 1649, chez E. Hébert (90 occurrences) ; L'On du temps tout nouveau, en vers burlesque (sic), par C. D. B. M., 1649 (7 occurrences).

50 Dans le seul L'On du temps tout nouveau, en vers burlesque (sic), par C. D. B. M.

51 Parmi les 3452 occurrences de « on » dans le corpus BurLesque Fronde, 115 sont en capitales ( $\mathrm{ON} »)$, soit $6 \%$.

52 Le pronom en capitales est présent dès la page de titre, inséré dans une vignette décorative : https://mazarinum.bibliotheque-mazarine.fr/expositions-virtuelles/item/17782-iiformes-et-genres-alternatifs-manuscrits-placards-et-chansons?oeuvre $=8$.

53 Agréable récit de ce qui s'est passé aux dernières barricades de Paris, décrites en vers burlesques, p. 13.

54 Pour des exemples caractéristiques, voir : le Premier courrier françois, traduit fidèlement en vers burlesques (C. Boudeville, 1649), le Courrier burlesque de la guerre de Paris, envoyé à monseigneur le prince de Condé, pour divertir Son Altesse durant sa prison : ensemble tout ce qui se passa jusqu'au retour de Leurs Majestés (1650) et le Courrier burlesque de la paix de Paris (1649).

55 "Quand le peuple sera pour nous / Sans doute qu'on filera doux", p. 7 ; de fait on attribue généralement cet écrit à un familier de Gaston d'Orléans, du côté de la Cour à cette date.

56 http://atilf.atilf.fr.

57 Ces rimes sont d'ailleurs utilisées comme contraintes de poèmes monorimes (L'envoy de Mazarin au Mont Gibel, ou l'étique de Mazarin, 1649, rimé entièrement sur "-ique ») ou birimes (Virelay sur les vertus de Sa Faquinance, 1652, rimant sur "-if " et "-aire " exclusivement).

58 Cette expression condensée inclut les différents types de bruit (dus à la segmentation ou à la transcription) et les silences. 
59105 textes ont été transcrits manuellement, parmi cet ensemble : https://github.com/Antonomaz/Corpus/tree/main/Mazarinades/.

60 Voir https:/github.com/Antonomaz/Corpus/tree/main/Mazarinades/.

61 Voir note 16 et http://antonomaz.huma-num.fr/tools/Biblio_Moreau.html.

\section{Auteurs}

\section{Karine Abiven}

EA 4509 STIH, Sorbonne Université, Paris, France

Karine Abiven est maîtresse de conférences en langue et littérature françaises. Elle travaille sur les écrits à large diffusion au XvII ${ }^{\mathrm{e}}$ siècle, en particulier polémiques, sous l'angle du style et des genres de discours.

ORCID 0000-0001-9518-1040

karine.abiven@sorbonne-universite.fr

\section{Jean-Baptiste Tanguy}

EA 4509 STIH, Sorbonne Université, Paris, France

Jean-Baptiste Tanguy est doctorant en humanités numériques. Il s'intéresse spécifiquement aux données bruitées et à leur usage pluriel et varié en humanités numériques.

ORCID 0000-0002-0007-1664

jean-baptiste.tanguy@sorbonne-universite.fr

\section{Gaël Lejeune}

EA 4509 STIH, Sorbonne Université, Paris, France

Gaël Lejeune est maître de conférences en informatique, spécialisé en traitement automatique des langues (TAL). Il s'intéresse à la variation dans les données (langue, genre, registre, bruit, etc.) et à son influence sur l'efficacité des systèmes de TAL (classification, extraction d'information, étiquetage, etc.).

ORCID 0000-0002-4795-2362

gael.lejeune@sorbonne-universite.fr

\section{Droits d'auteur}

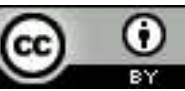

Les contenus de la revue Humanités numériques sont mis à disposition selon les termes de la Licence Creative Commons Attribution 4.0 International. 